\title{
A Influência dos Sistemas Integrados no Gerenciamento do Desempenho em Instituições Públicas
}

\author{
Gesualdo Menezes Cavalcante \\ Mestrado em Controladoria pela Universidade Federal Rural de Pernambuco - UFRPE \\ Servidor Público Federal lotado no Instituto Federal de Educação, Ciências e \\ Tecnologia de Alagoas - IFAL \\ Professor do Centro Universitário CESMAC - Campus IV \\ Rua prof. Ângelo Neto, 51. Farol. Maceio/AL. CEP: 57.051-530 \\ E-mail: gesualdom@hotmail.com \\ João Inocêncio Junior
Mestrado em andamento em Ciências Contábeis pela Universidade Federal de
Pernambuco - UFPE
Rua Conselheiro Portela, 665, Sala 119. Espinheiro. Recife/PE. CEP: $52.020-035$
E-mail: joaoinocenciojr@gmail.com
}

Alessandra Carla Ceolin Doutorado e Pós-doutorado em Agronegócios pela Universidade Federal do Rio Grande do Sul - UFRGS Professora na Universidade Federal Rural de Pernambuco - UFRPE Rua Dom Manuel de Medeiros, s/n. Dois Irmãos. Recife/PE. CEP: 52.171-900

E-mail: alessandra.acc@gmail.com

\section{RESUMO}

Este estudo buscou verificar se o Sistema Integrado de Patrimônio, Administração e Contratos (SIPAC) trouxe um aumento de desempenho no trabalho para os servidores do Instituto Federal de Alagoas (IFAL). Quanto aos aspectos metodológicos, realizou-se uma pesquisa exploratória e descritiva e de abordagem mista. Para a análise dos resultados, utilizou-se estatística descritiva e inferencial, além da análise de conteúdo. Os resultados descritivos apontaram que o sistema SIPAC trouxe um aumento de desempenho no trabalho dos servidores, apresentando um percentual de aumento acima de $60 \%$ em três dos quatro quesitos analisados. Os testes de Mann Whitney, considerando $\mathrm{P}<0,05$ de significância, evidenciaram que servidores com maior tempo de utilização com o sistema SIPAC possuem maior facilidade na sua operação; utilizamno com maior rapidez e tem maior produtividade no trabalho. Na visão dos gestores, os maiores benefícios apontados são agilidade na realização do trabalho e na localização dos processos, aumento da produtividade, confiabilidade e segurança nos controles internos.

Palavras-chave: Sistema Integrado de Gestão. UTAUT. Desempenho. 


\section{The Influence of Integrated Systems on Performance Management in Public} Institutions

\section{ABSTRACT}

This study sought to verify if the Integrated System of Patrimony, Administration and Contracts (SIPAC) increases work performance to the public employees (from the Federal Institute of Alagoas) (IFAL). Regarding the methodological aspects, exploratory and descriptive research was carried with a mixed approach. For the result analysis, a descriptive and inferential statistic was used on the content analysis. The descriptive results indicated that the SIPAC system brought an increase in employees work performance, presenting an increase percentage above $60 \%$ in three of the four items analyzed. In Mann Whitney's tests, considering $P<0.05$ of significance, evidenced that servers with longer usage time with the SIPAC system have easier operation abilities, use it faster and they have greater productivity at work. In the view of managers, the biggest benefits pointed out are agility in carrying out the work and in the localization of internal procedures. It increased: productivity, reliability and safety in the internal controls.

Keywords: Integrated Management System. UTAUT. Performance.

\section{INTRODUÇÃO}

A Tecnologia da Informação ( $\mathrm{Tl}$ ) vem mudando de forma crescente e significativa a maneira de trabalho e gestão das organizações com o uso dos Sistemas de Informações (SIs). Esse crescimento justifica-se pelo fato de que a TI proporciona melhorias importantes nos processos, pois pode tornar os processos gerenciais mais eficazes (O'Brien, 2010). Batista (2012) pontua que os Sls servem para melhorar os fluxos de informações e gerar relatórios eficazes para a tomada de decisão em todos os níveis organizacionais.

Dentre as categorias de Sls, os sistemas integrados de gestão ou Enterprise Resource Planning (ERPs) estão entre os mais utilizados, pois integram todos os processos da instituição em um só sistema (Batista, 2012). Quando se trata dos serviços públicos, o potencial das mudanças na administração pública por meio da TI se associa a vários paradigmas que norteiam as experiências de reforma e inovação no Brasil (Fernandes, 2004). Rabenschlag, Roratto e Dias (2012) destacam que, nos 
últimos anos, os projetos de TI nas entidades do setor público vêm ganhando foco nos investimentos devido às vantagens competitivas proporcionadas pelos Sls.

A implantação dos ERPs nas instituições públicas vem mudando significativamente a maneira de trabalhar dos servidores, pois a instituição passa a ter acesso e controle sobre as informações e atividades, provocando um dinamismo e a diminuição do tempo na execução das rotinas administrativas (Elihimas, 2015). Entre as instituições que vêm utilizando os sistemas ERPs, estão os Institutos Federais (IFS), que buscam um mecanismo de modernização, possibilitando a integração das atividades administrativas e o aumento do controle, além de fornecerem uma maior efetividade na tomada de decisão para os gestores públicos (Medeiros Júnior, 2014; Vecchia, 2011).

Neste sentido, diante da necessidade constante de informações para controle e gestão, em 2012, o Instituto Federal de Educação, Ciências e Tecnologia de Alagoas (IFAL) firmou um termo de cooperação com a Universidade Federal do Rio Grande do Norte (UFRN), no qual o IFAL passou a utilizar os sistemas desenvolvidos por essa instituição, incluindo o Sistema Integrado de Patrimônio, Administração e Contratos (SIPAC), objeto deste estudo.

O SIPAC é um ERP que serve para complementar e ampliar o controle interno das instituições, pois, além de realizar o controle físico, com valores unitários e totais dos bens de consumo e patrimoniais, também disponibiliza diversos dispositivos que auxiliam o controle nas atividades administrativas, de ensino, pesquisa e extensão (Medeiros Júnior, 2014; Souza, 2016). De acordo com SINFO (2017), o número de instituições públicas que utilizam o SIPAC no Brasil vem aumentando a cada ano, totalizando 61 organizações distribuídas em todo o país. Tal prática colaborou com o Plano Diretor da Reforma do Estado em 1995; já delineara a necessidade do Princípio da Eficiência na Constituição (Di Pietro,1999). Nesse sentido, menciona-se, an passant, que o conceito de eficiência é econômico e não jurídico e orienta a atividade administrativa dos órgãos, objetos da pesquisa buscar ferramentas necessárias à modernização. 
Apesar dos avanços proporcionados pela TI, Venkatesh, Morris e Davis (2003) destacaram que, para auferir os benefícios e resultados almejados com os investimentos, é preciso que as tecnologias sejam aceitas e utilizadas pelos seus usuários. Nesse sentido, os autores criaram a Unified Theory of Acceptance and Use of Technology (UTAUT), (em português, a Teoria Unificada de Aceitação e Uso da Tecnologia) e é a Teoria considerada Estado da Arte em aceitação e uso de tecnologias (Mariano \& Dias, 2017).

Segundo Venkatesh et al. (2003), o modelo UTAUT pode explicar as intenções dos usuários para utilizar um sistema e seu comportamento posterior. O modelo é baseado em quatro construtos determinantes, que são moderados pelos construtos moderadores. Os autores destacam que o construto expectativa de desempenho é o fator que mais influencia a intenção de uso da TI. Diante do exposto, os objetivos desta pesquisa são verificar se o sistema SIPAC trouxe um aumento de desempenho no trabalho para os servidores no IFAL e examinar a influência da expectativa de desempenho na aceitação e uso do sistema SIPAC no IFAL.

\section{TEORIA UNIFICADA DE ACEITAÇÃO E USO DA TECNOLOGIA (UTAUT)}

Vários modelos sugiram a partir da década de 1970 e foram aperfeiçoados ao longo dos anos com a intenção de estabelecer novos fatores que comprovassem a aceitação e o uso de uma tecnologia (Löbler, Visentini, \& Vieira, 2010). Dentre os modelos que surgiram, destacam-se: Theory of Reasoned Action (TRA) - Teoria da Ação Racionalizada de Fishbein e Azjen (1975), Technology Acceptance Model (TAM) Modelo de Aceitação de Tecnologia de Davis, Bagozzi e Warshaw (1989), Extension Technology Acceptance Model (TAM2) - Extensão do Modelo de Aceitação de Tecnologia de Venkatesh e Davis (2000), dentre outros.

A adoção de uma nova tecnologia torna-se interessante para a previsão de futuras tendências, possibilitando que as organizações obtenham economia financeira e possam conduzir pesquisas sobre produtos e/ou serviços que poderão ter uma maior 
probabilidade de aceitação perante seus usuários (Patrakosol \& Olson, 2007). A adoção está associada à forma com que os usuários enfrentam uma novidade e relacionada com o processo de dispersão pela qual uma inovação é disseminada aos indivíduos, e estes respondem com aceitação ou rejeição (Rogers, 1995).

Segundo Bautzer (2009), a forma como as pessoas reagem ao lançamento de uma nova tecnologia interessa às organizações. Venkatesh et al. (2003) pontuam que apenas o uso de uma nova tecnologia não significa que existe uma melhora no desempenho ou na produtividade, sendo fundamental que haja uma concreta utilização e aceitação dessa tecnologia por parte de seus usuários.

Diehl (2012) destaca que a aceitação e o uso da TI tem sido extensivamente investigada ao longo das últimas duas décadas, como nos estudos de Davis (1989 e 1993), Venkatesh e Davis (1996 e 2000), Venkatesh et al. (2003), Davis e Venkatesh (2004), Venkatesh e Bala (2008). A utilização de modelos originários da psicologia, sociologia e SI que muitas vezes eram utilizados isoladamente, foram agrupados por Venkatesh et al. (2003), a fim de buscar compreender o comportamento dos indivíduos frente ao surgimento e utilização de novas tecnologias (Oliveira, 2011).

Criou-se a UTAUT a fim de medir o sucesso na inserção de novas tecnologias nas organizações, compreender as intenções do usuário para manusear um determinado SI e prever tendências de usos subsequentes. O modelo UTAUT foi baseado na compilação de 8 modelos e 32 construtos influentes na aceitação de tecnologia (Figura 1). 


\begin{tabular}{|l|l|}
\hline \multicolumn{1}{|c|}{ Modelo/Teoria } & \multicolumn{1}{c|}{ Autores } \\
\hline Theory of Reaseoned Action (TRA) & Fishbein e Ajzen (1975) \\
\hline Tecnology Acceptance Model (TAM) & Davis (1985) \\
\hline Social Cognitive Theory (SCT) & Bandura (1986) \\
\hline Model of PC Utilization (MPCU) & Thompson, Higgins e Howell (1991) \\
\hline Theory of Planned Behavior (TPB) & Ajzen (1991) \\
\hline Innovation Diffusion Theory (IDT) & Rogers (1995) \\
\hline TAM e TPB combinados & Taylor e Todd (1995) \\
\hline Motivational Model (MM) & Vallerand (1997) \\
\hline
\end{tabular}

Figura 1. Modelos e Teorias Constituintes do UTAUT

Fonte: Adaptado de Venkatesh et al. (2003).

Fontes de outras áreas da ciência contribuíram significativamente para a construção do modelo (Costa, Castro, \& Cappellozza, 2014; Silva, 2009). Siswanto Shofiati e Hartini (2017) reforçam que a UTAUT é basicamente o Estado da Arte.

A UTAUT é baseada em quatro construtos determinantes: expectativa de desempenho, expectativa de esforço, influência social e condições facilitadoras. Ainda se definem os construtos moderadores da intenção de uso e do uso efetivo de tecnologias a saber: gênero, idade, experiência e voluntariedade de uso por parte dos usuários.

O modelo UTAUT considerou a influência dos construtos moderadores para cada um dos construtos determinantes, e concluíram que os construtos expectativa de desempenho, expectativa de esforço e influência social influenciam diretamente a "intenção de uso" de um sistema; já as condições facilitadoras influenciam diretamente o "efetivo uso" de um sistema. Ressalte-se que os quatro construtos determinantes decorrem da supressão de outros principais e intermediários nas diferentes teorias desenvolvidas por três décadas (Venkatesh et al., 2003).

Para análises dos construtos presentes na teoria, elaborou-se para melhor visualização a Figura 2. 


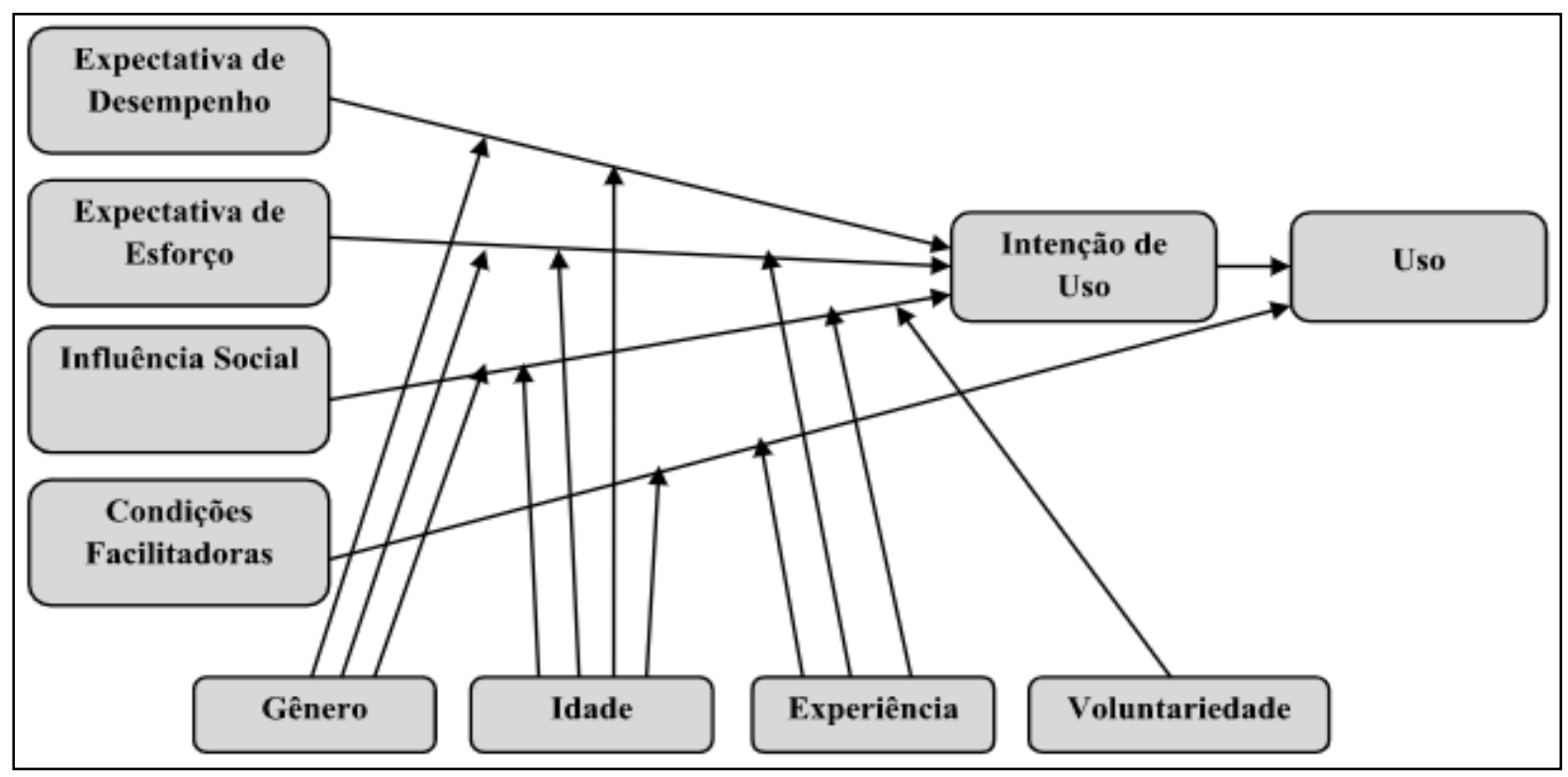

Figura 2. Teoria Unificada de Aceitação e Uso de Tecnologia (UTAUT) Fonte: Venkatesh et al. (2003).

O construto expectativa de desempenho é formado por seis principais variáveis: utilidade percebida, motivação extrínseca, adaptação ao trabalho, vantagem relativa, expectativa de resultado e facilidade de uso percebida. Já o construto expectativa de esforço compõe-se de três variáveis básicas: facilidade de uso percebida, complexidade e facilidade de uso. A influência social também é formada por mais três construtos base: norma subjetiva, fatores sociais e imagem; e as condições facilitadoras, assim como os outros dois construtos anteriores, também possuem como sua formação principal três variáveis principais: percepção de controle comportamental, condições facilitadoras e comparabilidade (Venkatesh et al., 2003). Frise-se que os quatro construtos determinantes da UTAUT possuem outros construtos na sua formação; entretanto, aqui colecionam-se os mais relevantes.

Na pesquisa, observou-se a influência das variáveis moderadoras (gênero, idade, experiência e voluntariedade) em cada construto (expectativa de desempenho, expectativa de esforço, influência social e as condições facilitadoras). Destaque-se ser a "experiência" (variável moderadora) referente ao prévio contato e familiaridade com 
outras tecnologias e a "voluntariedade" relacionada à crença de que o uso de sistemas é compulsório no ambiente laboral. O construto condições facilitadoras tem influência direta sobre o efetivo uso de uma tecnologia. Já os construtos expectativa de desempenho, expectativa de esforço e influência social têm influências: direta na intenção de uso e indireta no efetivo uso de tecnologias.

O modelo UTAUT pode auxiliar gestores, organizações e empresas desenvolvedoras de software que precisem avaliar a possibilidade de sucesso de uma nova tecnologia, determinando os fatores direcionadores da aceitação e do uso dos mesmos (Perácio, 2017). Outrossim, é modelo utilizado em vários países para verificar a intenção de uso de SI em diferentes tipos de usuários e tecnologias. Utilizou-se, na avaliação de ferramenta de ensino a distância na Arábia Saudita (Al-Gahtani, 2001), nos sistemas de voto eletrônico nos Estados Unidos (Yurong \& Marphy, 2007) e em aplicativos de escritórios na China (Chiu \& Wang, 2008), dentre outros.

A UTAUT é excelente ferramenta destinada aos Gestores que decidam implantar novas tecnologias, uma vez que os resultados de sua aplicação são satisfatórios em $70 \%$ da variância de intenção de uso e uso real de uma tecnologia a partir de determinados construtos. Diante do exposto, A UTAUT será utilizada nesta pesquisa devido à sua relevância e completude. Excepcione-se a "voluntariedade" (variável moderadora), cuja aplicação não é considerada neste estudo porque o SIPAC é de uso compulsório (Cavalcante, Ceolin, \& Barros, 2018).

\section{METODOLOGIA}

Para atingir o objetivo geral desta pesquisa, foi utilizado o procedimento técnico do estudo de caso. O SIPAC é um sistema integrado de gestão, criado pela UFRN, que possui diversas funcionalidades direcionadas às atividades administrativas e complementa e amplia o controle interno. Realiza o controle físico (com valores unitários e totais dos bens de consumo e patrimoniais) e disponibiliza dispositivos que auxiliam o controle efetuado pelo Sistema Integrado de Administração Financeira do 
Governo Federal (SIAFI) na gestão patrimonial, financeira e contratual (Silva, 2012; Sousa, 2016). O Estudo de Caso é estratégia valiosa em pesquisas de SI, pois viabiliza a interpretação das relações existentes entre os componentes de TI e os indivíduos no ambiente organizacional (Medeiros Júnior, 2014).

Quanto aos objetivos, a pesquisa é exploratória e descritiva, pois é utilizada quando o assunto abordado é pouco explorado e se pretende aprofundar 0 conhecimento. Já a pesquisa descritiva busca investigar determinado fenômeno e descrever suas características de forma como ocorrem, sem a interferência do pesquisado. Já em relação à abordagem, este estudo é misto (quali-quantitativo) (Michel, 2005; Borinelli, 2006).

Para fins de coleta de dados, foi aplicado um questionário junto aos servidores e realizadas entrevistas semiestruturadas com os gestores, todos usuários do SIPAC. O questionário, assim como o roteiro de entrevista fora adaptado da UTAUT. Para o questionário, utilizou-se a escala de concordância do tipo Likert de 5 pontos, onde foi atribuído 1 ao "discordo totalmente" e 5 ao "concordo totalmente". 0 instrumento foi dividido em 2 blocos: o primeiro teve 6 perguntas, sendo 4 relacionadas aos construtos moderadores (gênero, idade, experiência com SI e o SIPAC), além da escolaridade e o grupo/diretoria de atuação; e o segundo bloco teve 16 perguntas relacionadas à UTAUT, e para esse artigo, foi utilizado o recorte de questões referentes ao construto expectativa de desempenho.

A escala de concordância do tipo Likert apresenta uma grande contribuição em estudos que envolvam pesquisa de opinião justamente por evitar respostas binárias; ao contrário, busca uma adequação do estímulo de concordância psicológica ao sujeito cuja opinião é objeto de pesquisa. Em outros trabalhos das mais diversas instituições, é um lugar comum (Souza \& Durigon, 2014).

Os grupos moderadores (gênero, idade, experiência com SI e com sistema SIPAC) foram submetidos a testes de diferença de média que, de acordo com o modelo UTAUT, devem apresentar níveis diferentes de desempenho. Além dos grupos moderadores, também foi testada a escolaridade, tendo em vista que se trata de uma 
Instituição de Ensino Superior (IES). A hipótese é de que os servidores com maior instrução poderiam obter um maior desempenho com a utilização do sistema, bem como os três grupos que compõem a Pró-Reitoria de Administração do IFAL, com o intuito de verificar se há diferenças significativas de desempenho nesses grupos. Ressalta-se que os três grupos em que se dividiu a Pró-Reitoria de Administração (PROAD) neste estudo foram: Diretoria de Orçamento e Finanças (DOF), Diretoria de Suprimentos (DS) e na lotação chamada de "Subordinados à Coordenação e Diretamente ao Pró-Reitor" (SEMD), ou seja, os servidores são subordinados diretamente ao coordenador ou ao Pró-Reitor de Administração sem uma diretoria específica.

Foram comparadas as variáveis do bloco 1 em relação às 4 questões do construto expectativa de desempenho retiradas do modelo UTAUT, onde foram procedidos os testes de diferença de média não paramétrica, considerando a não normalidade da amostra de Mann Whitney $(\mathrm{U})$ nos casos de 2 amostras independentes (gênero, idade, escolaridade, experiência com SI e com sistema SIPAC) e o teste de Kruskal Wallis, no caso assumido de 3 amostras distintas (diretoria/grupo de atuação). Foram consideradas para análise, neste estudo, as médias cujos níveis de significância foram iguais ou inferiores a $5 \%(p \leq 0,05)$.

Pondera-se que, para fins de comparação neste trabalho, a variável faixa etária foi dividida em grupos de até 40 anos e mais de 40 anos; a escolaridade foi classificada em dois grupos, onde o primeiro possuía membros de Ensino Médio e Curso Superior completo, ou mesmo, cursando MBA/Especialização. No segundo, foram incluídos os servidores que terminaram MBA/Especialização ou possuem pós-graduação stricto sensu. A experiência com Sistemas de Informação (SI) foi dividida em um grupo com até 5 anos, e outro acima de 5 anos, enquanto que a experiência com SIPAC foi dividida em grupos de até 3 anos e acima de 3 anos, considerando que, no período da pesquisa, o sistema possuía pouco mais de 5 anos de implantação.

A validação dos instrumentos foi realizada por servidores/usuários do IFAL de outras unidades. Uma vez aprovados, os questionários e entrevistas foram aplicados in 
loco, no mês de novembro de 2017, a 41 servidores e 5 gestores respectivamente lotados na PROAD, da unidade da Reitoria. A amostra obteve um total de $93,8 \%$ do total de servidores lotados na PROAD, sendo 29 do sexo masculino, 27 até 40 anos, 34 com pós-graduação concluída (lato ou stricto sensu) e, quanto à lotação, 17 pertenciam à DOF: 17 à DS e 12 à SEMD.

Para as análises dos questionários, utilizaram-se estatísticas descritivas por meio do software Microsoft Excel, versão 2007, e análises inferenciais foram obtidas por meio do software Statistical Package for the Social Sciences (SPSS), versão 12.0.

Já para as interpretações das entrevistas, foi utilizada a técnica de análise de conteúdo, utilizando-se as três fases descritas por Bardin (2006): (i) pré-análise, onde os dados brutos foram colhidos sem recorte ou qualquer interferência dos autores; (ii) exploração do material em que se fez o recorte e as transcrições de trechos essenciais, enxugamento dos dados brutos; e (iii) tratamento dos resultados, onde foram feitas interpretações, análises reflexivas e críticas das entrevistas.

\section{ANÁLISE E DISCUSSÃO DOS RESULTADOS}

\subsection{Análises e Discussão das Entrevistas}

Com o intuito de verificar a opinião dos gestores em relação ao construto "expectativa de desempenho", foram apresentadas 4 questões distintas: a primeira relacionada ao impacto que o SIPAC trouxe nos hábitos de trabalhos dos usuários; a segunda está associada ao aumento/diminuição que o SIPAC trouxe na produtividade dos usuários; a terceira visa conhecer se o SIPAC pode ser visto como uma ferramenta que ajuda o servidor a obter reconhecimento e/ou promoções; e quarta e última almeja saber se os usuários reconhecem a utilidade do SIPAC.

Em relação à primeira questão, todos os entrevistados informaram que o sistema SIPAC tem mudado de forma positiva os hábitos de trabalho dos servidores, pois o sistema deu maior celeridade, agilidade e confiabilidade nos processos e controles da instituição. O entrevistado A citou como exemplo de confiabilidade o caso 
prático de que, com a presença do SIPAC, a instituição passou a ter um sistema de memorando que deixa tudo registrado. No entanto, fez questão de informar que, no início, houve resistência para a implantação do sistema, admitindo que: "[...] claro que no início da utilização, o servidor pode ter um pouco de resistência, mas uma resistência natural, não de rejeição, depois eles vão se adequando e também vão sendo críticos [...]".

Os entrevistados B e C pontuaram sobre os benefícios no aumento do desempenho no trabalho dos servidores que o sistema trouxe para a instituição. O entrevistado B cita o fato de que, com o SIPAC, "[...] você pode trabalhar ou obter informação de qualquer lugar - em casa, na rua, pelo celular [...]". Já o entrevistado C ressalta o impacto positivo nos hábitos de trabalho: "[...] é diferente de usar planilha do Excel e outros controles manuais que não têm uma confiabilidade nas informações; muitas vezes aconteciam erros [...]".

$O$ entrevistado $D$ reconhece que o sistema melhora e que veio para melhorar os hábitos de trabalho, os fluxos de processo e os controles da instituição. No entanto, relata que existe uma deficiência porque o seu departamento ainda não tem todos esses controles, e:

[...] ainda existe o problema do ser humano. É que as pessoas ainda não saíram da forma antiga de trabalho e não estão conseguindo visualizar a utilização do sistema. Têm módulos que ainda não conseguimos implantar integralmente porque algumas pessoas ainda não se desvincularam dos sistemas antigos [...].

Esse relato deixa implícito que pode haver problema de resistência dos servidores em aceitar e utilizar o SIPAC, como também problemas culturais que estão impedindo a completude da implantação. Continuando, informou que está faltando uma atitude ou uma determinação superior que obrigue a implantação do sistema: "[...] eu acho que se a gente for dando espaço às pessoas e for prorrogando a implantação do sistema, a gente nunca vai conseguir implantar [...]". 
No que diz respeito à segunda questão, em sua totalidade, os entrevistados afirmaram que o uso do SIPAC influencia diretamente e positivamente na produtividade, ou seja, o sistema melhora a produtividade de seus usuários. Neste sentido, o entrevistado A citou como exemplo de melhoria da produtividade um caso prático que ocorreu no setor de almoxarifado e projeções de melhorias futuras com módulos de compra e orçamento:

[...] o controle do almoxarifado em 2010 era manual, tudo ficha cardex, a gente não tinha condições de fazer aquela curva $A B C$, não tinha condições de fazer o estoque mínimo... e agora com o SIPAC, a gente consegue fazer. E vamos fazer mais quando o módulo de compras estiver integrado como módulo de orçamento [...].

Continuando seu depoimento, o entrevistado A relatou que faz uso de controles paralelos: "[...] ainda hoje, todos os contratos do IFAL são controlados através de planilhas eletrônicas, que podem ser falhas; você pode cometer equívocos [...]". Ressalta-se que o entrevistado $E$ informou que, antes do SIPAC, também usava planilhas eletrônicas e que não era seguro. $O$ entrevistado $A$ ainda pontuou que, com a implantação dos SIPAC, os controles são mais confiáveis, pois os usuários não vão precisar estar alterando as planilhas e mexendo em fórmulas, pois com o sistema, os cálculos são automáticos.

Já o entrevistado B informou que o SIPAC melhora a produtividade, todavia fez considerações importantes sobre pontos de melhoria, os quais podem ser considerados como fatores de resistência ao uso do sistema: "[...] o SIPAC precisa melhorar a questão visual, de interface com o usuário. É muito poluído e com informações desnecessárias, precisa de customização [...]". Inclusive, deixou transparecer que o atraso na customização pode estar relacionado à falta de atitude dos usuários, ao salientar que "[...] é preciso que os setores apontem as necessidades de melhorias e passem esta demanda. Existe, inclusive, um contrato com uma empresa para fazer a customização quando é demandada; esta empresa está disponível para isso [...]. 
O entrevistado $C$ pontuou que não existem dúvidas sobre o ganho de produtividade que os setores que já implantaram o SIPAC obtiveram, mas que ainda não consegue mensurar esse ganho em termos quantitativos: "[...] em todos os módulos que foram implantados, o ganho de produtividade foi muito grande, o que fez com que a gente direcionasse esforços para outras atividades que antes não conseguíamos fazer [...]". Corroborando, o entrevistado E ratificou que, com o SIPAC, "[...] a produtividade aumenta, pois não há perda de tempo na localização do processo, coisa que antes do sistema era muito normal acontecer. Antes, o protocolo era manual e a gente tinha uma dificuldade de achar um processo [...]".

Nas descrições referentes à terceira questão, de acordo com o entrevistado $A$, a instituição possui critérios bem definidos para promoções e, em relação ao reconhecimento, discorreu que é observado o conjunto de atribuições do servidor no setor em que atua e não só pelo uso do SIPAC; e fez a seguinte colocação: "[...] eu vejo assim, para promoção ou reconhecimento eu não uso só da ferramenta SIPAC, não [...]". De forma semelhante, o entrevistado $B$ informou que o reconhecimento ou promoção do servidor não se dá propriamente pelo uso do SIPAC. Até reconheceu que o SIPAC pode ser utilizado como ferramenta para medir a produtividade dos servidores; entretanto, ressaltou que "[...] infelizmente, na grande maioria dos órgãos públicos que eu conheço não é esse o critério objetivo de ascensão e promoção [...]".

Já o entrevistado C respondeu que "[...] sim, com a ressalva que tem mais a ver com o perfil do servidor [...]". E complementou que os usuários do SIPAC aumentam as chances de obter reconhecimento ou promoções dentro da instituição. Na medida em que o servidor for dinâmico e procurar cada vez mais utilizar o sistema, ele vai aumentando a probabilidade de obter uma função gratificada, por exemplo. Similarmente, o entrevistado $D$ relatou que o reconhecimento ou promoção depende do esforço pessoal, que todo trabalho bem feito merece qualquer tipo de reconhecimento ou promoção; no entanto, deixou bem claro que o reconhecimento tanto pode ser por utilizar o SIPAC ou qualquer outro sistema. 
A resposta do entrevistado $\mathrm{E}$ foi ambígua, uma vez que, primeiramente, respondeu que não, e justificou que "[...] o uso do sistema é obrigatório dentro da instituição e não vai agregar nenhum tipo de promoção [...]". Depois, deixou implícito que, no contexto geral, a coordenação, para oferecer uma promoção, vai olhar para o conjunto das atribuições do servidor, e nesta perspectiva entraria o uso do SIPAC "[...] quando um gerente for olhar para a promoção, ele não vai olhar para o SIPAC e, sim, para o contexto como um todo. O sistema não é uma ferramenta que vai determinar a promoção ou não. E, sim, o conjunto das atribuições do servidor dentro do setor de protocolo [...]".

Referente à quarta questão deste construto, os entrevistados $A, B, C$ e E afirmaram que os usuários reconhecem a utilidade do sistema SIPAC; no entanto, o entrevistado $D$ relatou que a grande maioria dos usuários não reconhece a utilidade do sistema.

O entrevistado A afirmou que, apesar de a instituição ter o SIPAC há 3 ou 4 anos, ele ainda não está funcionando na sua totalidade, devido a vários problemas, mas, mesmo assim, os servidores reconhecem e buscam utilizar o SIPAC. Corroborando, o entrevistado B salientou "[...] não vejo como não reconhecer [...]", exemplificando com as melhorias obtidas pela instituição com o SIPAC nos módulos de protocolo e almoxarifado que, segundo ele, estão funcionando em sua plenitude: "[...] a gente não consegue mais trabalhar sem o SIPAC, seria um retrocesso se isso acontecesse $[\ldots]$ "..

Da mesma forma, o entrevistado $C$ afirmou que a utilidade do SIPAC é muito reconhecida dentro da instituição, e declarou que no início houve uma resistência natural por parte dos servidores em utilizar o sistema, mas que, com o passar do tempo, melhorou. Isso ficou evidente na sua fala: "[...] quando implantamos o memorando eletrônico, houve uma resistência inicial, mas hoje o reconhecimento das funcionalidades de que ele é algo bom existe, no geral [...]". Pactuando da mesma ideia, o entrevistado $E$ foi breve em sua fala, mas relatou acreditar que os usuários reconhecem "bem" a utilidade do sistema SIPAC. 
Contrapondo-se às afirmativas dos outros entrevistados, o entrevistado afirmou que a grande maioria dos usuários do sistema SIPAC não reconhece a sua utilidade. Informou, ainda, que falta um trabalho de demonstrar às pessoas a importância e as consequências de utilizar o sistema, e assim se expressa: "[...] às vezes as pessoas não sabem a importância do sistema e fazem as tarefas mecanicamente [...]". Este depoimento demonstra que podem ter existido falhas no processo de implantação do sistema, falta de comunicação ou envolvimento das pessoas que executam tarefas diárias.

\subsection{Análises Descritivas dos Questionários}

As Tabelas de 1 a 5 referem-se às análises descritivas dos questionários aplicados aos 41 servidores/usuários do sistema SIPAC no IFAL, lotados na PROAD. As questões foram adaptadas da UTAUT e referem-se ao Construto Expectativa de Desempenho, objeto deste trabalho.

Como se pode observar na Tabela 1, 65,8\% dos respondentes afirmam que, com o uso do SIPAC, conseguem terminar suas tarefas mais rapidamente. No entanto, verifica-se também que mais de um terço dos servidores $(34,2 \%)$, ou discordaram ou acham que o uso do sistema é imparcial, ou seja, não interfere no aumento da rapidez da execução de suas tarefas. Neste contexto, observa-se que a maioria dos respondentes corrobora os estudos de Costa, Lima e Souza Júnior (2017), em que foi constatado que o uso da $\mathrm{TI}$ nas organizações permitiu uma maior agilidade na execução das tarefas e, até mesmo, modificou a forma como são realizadas. 
A Influência dos Sistemas Integrados no Gerenciamento do Desempenho em Instituições Públicas

Gesualdo Menezes Cavalcante, João Inocêncio Junior, Alessandra Carla Ceolin

Tabela 1

Usar o sistema SIPAC me permite terminar minhas tarefas mais rapidamente

\begin{tabular}{lcc}
\hline \multicolumn{1}{c}{ Escala Likert } & Frequência & Percentual (\%) \\
\hline Discordo Totalmente & 2 & 4,9 \\
Discordo Parcialmente & 5 & 12,2 \\
Indiferente & 7 & 17,1 \\
Concordo Parcialmente & 21 & 51,2 \\
Concordo Totalmente & 6 & 14,6 \\
\hline Total & $\mathbf{4 1}$ & $\mathbf{1 0 0 , 0}$ \\
\hline
\end{tabular}

Nota. Fonte: Elaborada pelos autores.

De acordo com a Tabela 2, os percentuais dos respondentes que discordaram juntos com os que informaram que são indiferentes chegam a 68,3\%. Neste sentido, entende-se que o fato de os servidores usarem o sistema SIPAC não proporciona reconhecimento ou não é visto como forma de obter uma função gratificada, por exemplo. No entanto, destaca-se que $31,7 \%$ dos entrevistados concordaram que o uso do SIPAC aumenta as chances de obter promoções, pelo que se pode entender que esse reconhecimento ocorre em algum departamento ou diretoria.

Tabela 2

Se eu uso o sistema SIPAC, amplio minhas chances de obter um maior reconhecimento (mudança de função, participação em reuniões importantes, dentre outros) dentro do IFAL

\begin{tabular}{lcc}
\hline \multicolumn{1}{c}{ Escala Likert } & Frequência & Percentual (\%) \\
\hline Discordo Totalmente & 12 & 29,3 \\
Discordo Parcialmente & 4 & 9,7 \\
Indiferente & 12 & 29,3 \\
Concordo Parcialmente & 12 & 29,3 \\
Concordo Totalmente & 1 & 2,4 \\
\hline Total & $\mathbf{4 1}$ & $\mathbf{1 0 0 , 0}$ \\
\hline
\end{tabular}

Nota. Fonte: Elaborada pelos autores.

Constata-se, de acordo com os dados da Tabela 3, que o SIPAC é considerado, pela grande maioria dos respondentes $(92,7 \%)$, um sistema útil para a realização dos trabalhos dos servidores, e apenas $7,3 \%$ discordaram. Esses resultados podem 
evidenciar que a instituição tomou uma decisão correta ao adquirir o sistema como uma ferramenta que agrega valor às atividades de seus usuários.

Tabela 3

Eu acho que o sistema SIPAC é útil ao meu trabalho

\begin{tabular}{lcc}
\hline \multicolumn{1}{c}{ Escala Likert } & Frequência & Percentual (\%) \\
\hline Discordo Totalmente & - & - \\
Discordo Parcialmente & 3 & 7,3 \\
Indiferente & - & - \\
Concordo Parcialmente & 15 & 36,6 \\
Concordo Totalmente & 23 & 56,1 \\
\hline Total & $\mathbf{4 1}$ & $\mathbf{1 0 0 , 0}$ \\
\hline
\end{tabular}

Nota. Fonte: Elaborada pelos autores.

Ao se analisar a Tabela 4, a seguir, percebe-se que o SIPAC aumentou a produtividade de $61 \%$ dos respondentes, sinal de que o sistema está gerando resultados positivos no desempenho dos servidores, mas também cabe acrescentar que o restante dos servidores, ou seja, 39\% não observaram melhoras em seu desempenho com a utilização do sistema, o que sinaliza que esses servidores ainda não estão satisfeitos com a utilização do SIPAC. Calisir \& Calisir (2004) destacaram que os usuários finais de SI ficariam mais satisfeitos se acreditassem que a utilização do sistema cooperasse para o aumento do desempenho e da produtividade.

Tabela 4

Utilizar o sistema SIPAC aumenta minha produtividade

\begin{tabular}{lcc}
\hline \multicolumn{1}{c}{ Escala Likert } & Frequência & Percentual (\%) \\
\hline Discordo Totalmente & 5 & 12,2 \\
Discordo Parcialmente & 3 & 7,3 \\
Indiferente & 8 & 19,5 \\
Concordo Parcialmente & 14 & 34,2 \\
Concordo Totalmente & 11 & 26,8 \\
\hline Total & $\mathbf{4 1}$ & $\mathbf{1 0 0 , 0}$ \\
\hline
\end{tabular}

Nota. Fonte: Elaborada pelos autores. 
$\mathrm{Na}$ Tabela 5, são apresentados os somatórios dos resultados das análises descritivas sobre todas as questões do Construto Expectativa de Desempenho apresentados nas tabelas de 1 a 4 . Os resultados também estão distribuídos entre três grupos/diretorias distintos que pertencem à Pró-Reitoria de Administração: DOF, DS e SEMD. Destaca-se que, como o total de respondentes foram 41 e o número de questões foram 4, na Tabela 5 são apresentadas as 164 respostas possíveis para 0 construto expectativa de desempenho.

Tabela 5

Distribuição de respostas por Grupos/Diretorias que forma a PROAD

\begin{tabular}{|c|c|c|c|c|c|c|c|c|}
\hline \multirow{3}{*}{ Escala Likert } & \multirow{2}{*}{\multicolumn{2}{|c|}{$\begin{array}{c}\text { Diret. Orç. Finanças } \\
\text { Qt. Respostas }\end{array}$}} & \multirow{2}{*}{\multicolumn{2}{|c|}{$\begin{array}{c}\text { Diret. Suprimentos } \\
\text { Qt. Respostas }\end{array}$}} & \multirow{2}{*}{\multicolumn{2}{|c|}{$\begin{array}{l}\text { Sem Diretoria } \\
\text { Qt. Respostas }\end{array}$}} & \multirow{2}{*}{\multicolumn{2}{|c|}{$\begin{array}{c}\text { Total } \\
\text { Qt. } \\
\text { Respostas }\end{array}$}} \\
\hline & & & & & & & & \\
\hline & $\mathbf{N}$ & $\%$ & $\mathbf{N}$ & $\%$ & $\mathbf{N}$ & $\%$ & $\mathbf{N}$ & $\%$ \\
\hline \multirow{6}{*}{$\begin{array}{l}\text { Discordo Totalmente } \\
\text { Discordo } \\
\text { Parcialmente } \\
\text { Indiferente } \\
\text { Concordo } \\
\text { Parcialmente } \\
\text { Concordo Totalmente } \\
\text { Total }\end{array}$} & 19 & 31,7 & - & - & - & - & 19 & 11,6 \\
\hline & 14 & 23,3 & 1 & 1,6 & - & - & 15 & 9,1 \\
\hline & 14 & 23,3 & 13 & 20,3 & - & - & 27 & 16,5 \\
\hline & 13 & 21,7 & 36 & 56,2 & 13 & 32,5 & 62 & 37,8 \\
\hline & - & - & 14 & 21,9 & 27 & 67,5 & 41 & 25,0 \\
\hline & 60 & 100,0 & 64 & 100,0 & 40 & 100,0 & 164 & 100,0 \\
\hline
\end{tabular}

Nota. Fonte: Elaborada pelos autores.

A expectativa de desempenho pode ser definida como o grau em que um servidor público acredita que a utilização de um sistema vai contribuir para que ele aumente o desempenho na realização de suas atividades no trabalho, sendo o fator que mais influencia a intenção de uso da tecnologia da informação. Considerando os dados apresentados na Tabela 5, verifica-se que $62,8 \%$ das respostas para o construto expectativa de desempenho concordaram que o uso do sistema SIPAC possibilitou melhora no desempenho das atividades dos respondentes; entretanto, essa melhora não foi absoluta, visto que $37,8 \%$ das respostas concordaram parcialmente.

Já as análises separadas entre os três grupos/diretorias evidenciaram, como fator de maior relevância, o fato de que $100 \%$ dos respondentes lotados na SEMD afirmaram 
que obtiveram um aumento de desempenho com o uso do sistema SIPAC, pois $67,5 \%$ das respostas concordaram totalmente e $32,5 \%$ concordaram parcialmente. Já entre os servidores lotados na DS, $78,1 \%$ das respostas também sinalizaram um ganho importante no desempenho. Em contrapartida, entre os servidores que trabalham na DOF, apenas $21,7 \%$ das respostas concordaram parcialmente que o SIPAC produziu um aumento no desempenho, e a maioria das respostas (55\%) não concordam que o SIPAC trouxe melhoras no desempenho de suas atividades, revelando que existem fatores de resistências que precisam ser trabalhados para que a instituição possa colher um maior resultado com a utilização do sistema.

\subsection{Análises Inferenciais}

$\mathrm{Na}$ análise inferencial, foram feitas comparações entre cada uma das variáveis: gênero, faixa etária, escolaridade, grupo/diretorias de lotação, experiência na utilização com sistemas integrados e experiência na utilização do SIPAC com as questões referentes ao construto expectativa de desempenho da UTAUT. As comparações visaram testar se as variáveis moderadoras têm influência direta e indireta na intenção de uso e uso do sistema SIPAC com relação ao construto expectativa de desempenho. Não foram observadas diferenças significativas em relação à faixa etária, escolaridade, grupo/diretoria de lotação; no entanto, houve diferenças estatísticas significativas em relação à experiência com o sistema, conforme demonstra a Tabela 6. 
A Influência dos Sistemas Integrados no Gerenciamento do Desempenho em Instituições Públicas Gesualdo Menezes Cavalcante, João Inocêncio Junior, Alessandra Carla Ceolin

Tabela 6

Média e Desvio Padrão do Tempo de Utilização do SIPAC e Testes de Significância ( $P$-value)

\begin{tabular}{|c|c|c|c|c|c|}
\hline \multirow{2}{*}{ Variáveis } & \multicolumn{2}{|c|}{ Até 3 anos $(n=23)$} & \multicolumn{2}{|c|}{ Acima de 3 anos $(n=18)$} & \multirow[b]{2}{*}{$\boldsymbol{P}$} \\
\hline & Média $(\mu)$ & Desvio-padrão $(\sigma)$ & Média $(\mu)$ & Desvio-padrão $(\sigma)$ & \\
\hline $\begin{array}{l}\text { Q9 - Rapidez para terminar } \\
\text { tarefas }\end{array}$ & 3,26 & 1,01 & 4,00 & 0,97 & 0,016 \\
\hline $\begin{array}{l}\text { Q11 - Aumento das chances } \\
\text { de reconhecimento }\end{array}$ & 2,39 & 1,34 & 3,00 & 1,08 & 0,133 \\
\hline Q12- Útil ao trabalho & 4,13 & 0,97 & 4,78 & 0,43 & 0,010 \\
\hline $\begin{array}{l}\text { Q17 - Aumenta a minha } \\
\text { produtividade }\end{array}$ & 3,22 & 1,31 & 4,00 & 1,19 & 0,041 \\
\hline $\begin{array}{l}\text { Construto Expectativa de } \\
\text { Desempenho }\end{array}$ & 3,25 & 0,99 & 3,94 & 0,64 & 0,022 \\
\hline
\end{tabular}

Nota. Fonte: Elaborada pelos autores.

Nesse sentido, os resultados revelam que o aumento do desempenho dos servidores da Pró-Reitoria de Administração no IFAL, com o uso do sistema SIPAC, são mais perceptíveis para os usuários com o maior tempo de uso no sistema, o que corrobora o estudo de Davis et al. (1989), pontuando que o construto utilidade percebida está relacionado à crença de que um usuário possui que uma tecnologia vai melhorar sua produtividade em alguma tarefa no trabalho, e quanto maior for a utilidade percebida, maior será a intenção de adotar um comportamento.

Neste trabalho, optou-se por analisar a experiência do usuário sob duas óticas: um espectro geral de experiência com Sls e um mais específico sobre a experiência com o próprio sistema SIPAC dentro da instituição. Os resultados apontam que, diferentemente da experiência geral com Sls, no que concerne à experiência com o SIPAC, o construto expectativa de desempenho apresentou diferenças de média significativas a $5 \%$, conforme demonstra a Tabela 6 , embora o modelo UTAUT não prevê impactos da experiência. Em relação à experiência com o SIPAC, o construto expectativa de desempenho apresentou diferenças de média significativas. Usuários com experiência acima de 3 anos possuem uma melhor percepção em relação à rapidez para terminar as tarefas ( $\mu=4,00$, contra $\mu=3,26$ dos menos experientes), utilidade ao trabalho ( $\mu=4,78$, contra $\mu=4,13$ dos menos experientes), aumento da 
produtividade ( $\mu=4,00$, contra $\mu=3,22$ dos menos experientes) e em relação à média do próprio construto ( $\mu=3,94$, contra $\mu=3,25$ dos menos experientes).

\section{CONCLUSÕES}

Dificuldades quanto à baliza da pesquisa sempre vêm à tona, como a necessidade de escolher-se o modelo UTAUT com apoio da escala de tipo Likert, que é uma decisão que o encarregado tem de realizar quando da pesquisa, sendo no exterior o Estado da Arte.

Quanto aos objetivos da pesquisa, é, basicamente, analisar o SIG cujo nome é SIPAC, baseados na teoria UTAUT foram alcançados. Os principais resultados evidenciaram que, na visão dos gestores, acarretando maior otimização do tempo na realização dos trabalhos, maior rapidez na localização dos processos, aumento da produtividade, integração dos processos internos e também o fato de que o uso do sistema trouxe uma maior segurança para os controles internos: esses todos foram apontados como os maiores benefícios que o sistema SIPAC ofereceu à instituição.

Já do ponto de vista dos subordinados, a pesquisa foi satisfatória e indicou que o sistema SIPAC trouxe um aumento no desempenho de suas atividades.

Por fim, destaca-se que as informações colhidas neste estudo, além da relevância acadêmica, são relevantes aos gestores e servidores públicos que utilizam o SIPAC, para a própria UFRN (instituição que criou e atualiza os módulos do sistema), para o pessoal interno (suporte de $\mathrm{TI}$ ) e externo (empresas credenciadas para dar suporte nas diversas instituições públicas do Brasil), bem como para o entendimento, o aperfeiçoamento e a utilização da UTAUT em sistemas implantados em instituições públicas, as quais possuem particularidades.

No entanto, pontua-se que este trabalho foi realizado em uma Pró-Reitoria específica de uma instituição pública de ensino, não podendo ser generalizado. Dessa forma, recomenda-se que sejam feitas outras pesquisas relacionadas ao tema nos diversos órgãos e instituições que utilizam o sistema SIPAC. 


\section{REFERÊNCIAS}

Ajzen, I. (1991). The theory of planned behavior. Organizational Behavior and Human Decision Processes, 50(2), 179-211.

Al-Gahtani, S. (2001). The applicability of TAM outside North America: an empirical test in the United Kingdom. Information Resources Management Journal, 14(3), 37-46.

Bandura, A. (1986). Social foundations of thought and action: a social cognitive theory. Englewood Cliffs, Prentice Hall.

Bardin, L. (2006). Análise de conteúdo. Edições 70, Lisboa.

Batista, E. O. (2012). Sistema de Informação: o uso consciente da tecnologia para o gerenciamento. São Paulo, Saraiva.

Bautzer, D. (2009). Inovação: repensando as organizações. São Paulo, Atlas.

Borinelli, M. L. (2006). Estrutura Conceitual Básica de Controladoria: sistematização à luz da teoria e da práxis. (Tese de Doutorado). Universidade de São Paulo, USP, São Paulo.

Calisir, F., \& Calisir, F. (2004). The relation of interface usability characteristics, perceived usefulness, and perceived ease of use to end-user satisfaction with enterprise resource planning (ERP) systems. Computers in Human Behavior, 20(4), 505-515.

Cavalcante, G. M., Ceolin, A.C., \& Barros, J. E. M. (2018). Aceitação e Uso do Sistema Integrado de Patrimônio, Administração e Contratos (SIPAC) no Instituto Federal de Educação, Ciências e Tecnologia de Alagoas (IFAL): uma análise das diferenças de grupos. Revista Gestão Organizacional, 11, 79-96.

Chiu, C. M., \& Wang, E. T. G. (2008). Understanding web-based learning continuance intention - the role of subjective task. Information \& Management, 45(3), 194-201.

Costa, E. da. S., Castro, D. S. P. de., \& Cappellozza, A. (2014). Adoção e uso de portais de governo eletrônico no ambiente do sistema nacional de ciências e tecnologia e inovação: um estudo baseado no modelo UTAUT. Revista Eletrônica de Ciências Administrativa, 13(3). <http://www.periodicosibepes.org.br/index.php/recadm/article/view/1849>. 
Costa, T. F., Lima, D. F., \& Souza Júnior, A. M. (2017). Avaliação da usabilidade de sistema no modo clássico e drafiting e annotation do Autocad 2014. Revista Holos, 33(2).

Davis, F. D. (1985). A technology acceptance model for empirically testing new end user information systems: theory and results. Cambridge, MA. Doctoral Thesis. Massachusetts Institute of Technology, MIT.

Davis, F. D. (1989). Perceived usefulness, perceived ease of use, and user acceptance of information technology. MIS Quarterly, 13(3), 319-340.

Davis, F. D., \& Venkatesh, V. (2004) Toward preprototype user acceptance testing of new information systems: implications for software project management. IEEE Transactions on Engineering Management, 51(1) 31-46.

Davis, F. D., Bagozzi, R. P., \& Warshaw, P. R. (1989). User acceptance of computer technology: a comparison of two theoretical models. Management Science, 35(8), 982-1003.

Diehl, F. M. (2012). Um estudo de caso sobre a adaptação de usuários a mudanças de tecnologia da informação. (Dissertação de Mestrado). Pontifícia Universidade Católica, PUC, Porto Alegre, RS.

Di Pietro, M. S. Z. (1999). Direito Administrativo. São Paulo, Atlas.

Elihimas, J. C. M. (2015). Fatores críticos de sucesso na aderência de processos de negócio de ERP às organizações do setor público. (Dissertação de Mestrado). Universidade Federal de Pernambuco, UFPE, Recife, PE.

Fernandes, A. C. G. (2004). Algumas questões para o futuro. In: e-gov.br a próxima revolução brasileira. São Paulo, Prentice Hall, 2004.

Mariano, A. M., \& Dias, L. F. A. (2017). A importância da aceitação e uso da tecnologia em aplicativos de mobilidade urbana: contribuições da literatura científica. Anais do Congresso Brasileiro de Engenharia de Produção (ConBRepro). Ponta Grossa, PR, Brasil, 7.

Michel, M. H. (2005). Metodologia e pesquisa científica em ciências sociais. São Paulo, Atlas.

O’Brien, J. A. (2010). Sistemas de informação e as decisões gerenciais na era da Internet. São Paulo, Saraiva. 
Oliveira, B. M. K. de (2001). Aceitação e uso de ambiente virtual de aprendizagem no contexto de um curso de capacitação para servidores públicos. (Dissertação de Mestrado). Universidade Federal do Rio Grande do Norte, UFRN, Natal, RN.

Patrakosol, B., \& Olson, D. (2007). How interfirm collaboration benefits it innovation. Information \& Management, 44, 53-62.

Perácio, L. B. (2017). Inovação na Administração Pública: aplicação do modelo TAM/TTF para avaliação do sistema de controle de afastamento na UFMG. (Dissertação de Mestrado). Faculdade de Ciências Empresariais, FACE, Belo Horizonte, MG.

Rabenschlag, D. R., Roratto, R., \& Dias, E. V. (2012). Fatores de riscos no gerenciamento de projetos de tecnologia de informação no setor público brasileiro. Revista Espacios, 33(8). <http://www.revistaespacios.com/index.html>.

Rogers, E. M. (1995). Diffusion of Innovations. New York, The Free Press.

Silva, C. A. B. da. (2012). Arquitetura empresarial: um estudo de caso sobre a integração entre a plataforma MOODLE e o SIGAA na UFRN. (Dissertação de Mestrado). Universidade Federal do Rio Grande do Norte, UFRN, Natal, RN.

Silva, J. M. B. da. (2009). Aplicação do modelo UTAUT na avaliação de intenção de uso de sistemas ERP. (Dissertação de Mestrado). Faculdade de Economia e Finanças IBMEC, Rio de Janeiro, RJ.

Siswanto, T, Shofiati, R., \& Hartini, H. (2017, agosto, 9). "IOP Acceptance and Utilization of Technology (UTAUT) as a Method of Technology Acceptance Model of Mitigation Disaster Website". Conferência Earth and Environmental Science, 106(4). International Seminar on Sustainable Urban Development, Jakarta, Indonesia. Recuperado em 01 abril, 2020, de https://iopscience.iop.org/article/10.1088/17551315/106/1/012011.

Sousa, A. G. de. (2016). Design de interfaces e arquitetura da informação em sistemas de gestão acadêmica: redesign do SIPAC-UFRN. (Dissertação de Mestrado). Universidade Federal do Rio Grande do Norte, UFRN, Natal, RN.

Souza, M. A., \& Durigon A. R. (2014). Controladoria em Instituição pública de Ensino Superior: Percepção do seu papel sob a ótica dos gestores. Revista ABCustos, 9(1) 01-28.

Taylor, S., \& Todd, P. A. (1995) Assessing it usage: the role of prior experience. Mis Quarterly, 19(4) 561-570. 
Thompson, R. L., Higgins, C. A., \& Howell, J. M. (1991). Personal computing: toward a conceptual model of utilization. MIS Quarterly, 15(1) 124-143.

Vallerand, R. J. (1997). Toward a hierarchical model of intrinsic and extrinsic motivation. Advances in Experimental Social Psychology, 29, 271-360.

Vecchia, A. F. D. (2011). Sistemas ERP: a gestão do processo de implantação em universidade pública. (Dissertação de Mestrado). Universidade Federal de Santa Maria, UFSM, Santa Maria, RS.

Venkatesh, V., \& Bala, H. (2008). Technology acceptance model 3 and a research agenda on interventions. Decision Sciences, 39(2) 273-315.

Venkatesh, V., \& Davis, F. D. (2000). A Theoretical extension of the technology acceptance model: four longitudinal field studies. Management Science, 46(2) 186204.

Venkatesh, V., Morris, M. G., Davis, G. B., \& Davis, F. D. (2003). User acceptance of information technology: toward a unified view. Mis Quartely, 27(3) 425-478.

Yurong, Y., \& Murphy, L. (2007). Remote electronic voting systems: an exploration of voters perceptions and intention to use. European Journal of Information Systems, $16,106-120$.

Data de Submissão: 14/09/2019

Data de Aceite: 20/04/2020 